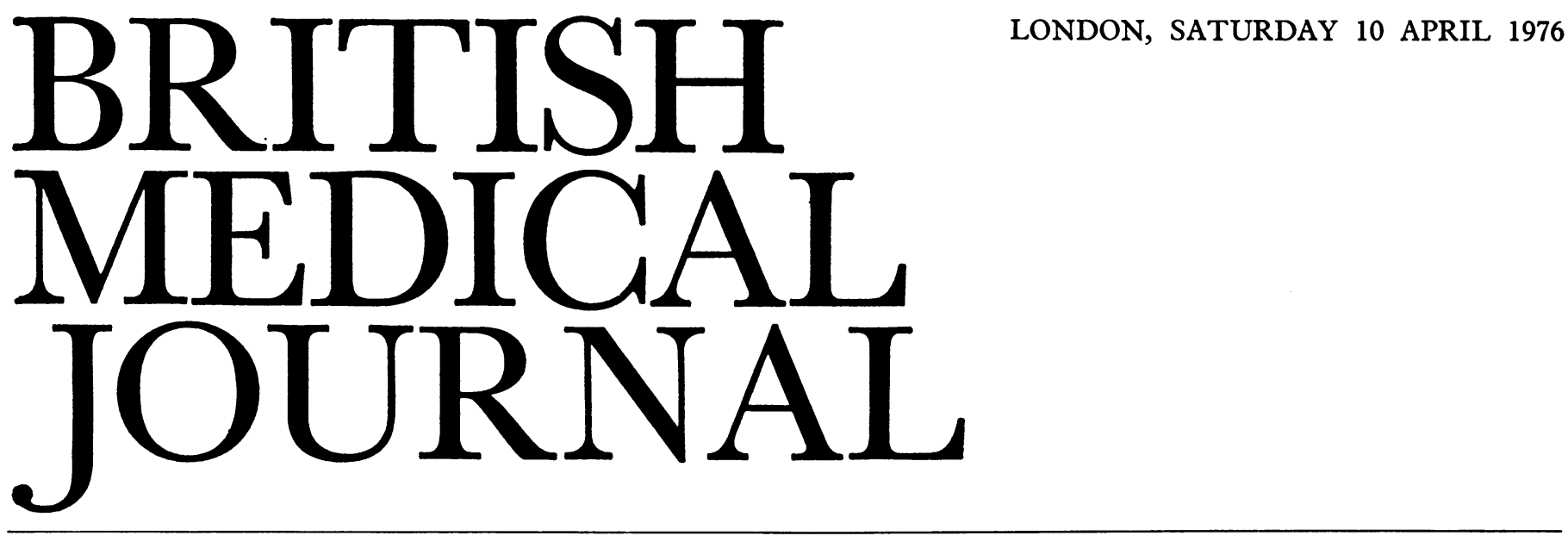

\title{
Prevention of coronary heart disease
}

Mortality from coronary heart disease has not been noticeably influenced by the expenditure of time, money, and manpower on coronary ambulances, coronary care, coronary surgery, and rehabilitation. The only ways we shall make any real impact on the disease will be to prevent it or to find a way of curing it. Cure is not only likely to be unattainable but would not help to prevent the sudden deaths that account for half the fatal cases unless screening for presymptomatic coronary disease could be made more successful and could be generally applied. ${ }^{1}$ Prevention is the better option, and it should be applied on nationwide rather than individual scales. We do not know the causes, still less the mechanisms, but we do know the associations of coronary disease and that most are remediable. The familial clustering of coronary disease is only rarely genetically determined; it is largely attributable to the sharing of family bad habits. Hard on the heels of the report of a joint working party of the Royal College of Physicians and the British Cardiac Society on the care of patients with coronary heart disease ${ }^{1}$ comes the report of their third working party on prevention, whose summary and recommendations are published at $\mathrm{p} 881$.

The report reviews the incriminating epidemiological evidence against high blood pressure, high blood fats, smoking, obesity, and physical inactivity. In the past, a theoretical and misleading distinction has been made between primary prevention (designed to avert symptomatic disease in symptomfree people) and secondary prevention (designed to prevent progression in people with recognised coronary disease), but the two are indivisible because so much life-threatening coronary heart disease is occult and unknown to both its future victim and his doctor. The report is therefore directed to general practitioners and is concerned with those risk-related traits which can be modified. The public health education necessary in order to achieve results could become a major concern of the new community physicians.

The saturated fat content of the diet is directly related to the height of the blood fats and to the incidence of death from coronary heart disease in the community. ${ }^{2}$ Populations which show very low mean serum cholesterol contents throughout life, such as Japan, also have a negligible incidence of coronary heart disease even when other risk markers such as high blood pressure, cigarette smoking, diabetes, urbanisation, and stress are common. That cholesterol is not all important in populations with higher than average cholesterols was shown in a comparative study of the incidence of coronary heart disease in Edinburgh and Stockholm. ${ }^{3}$ Deaths from this cause are two and a half times more common in Edinburgh men under the age of 60 than they are in Stockholm, though the mean cholesterol values in the two cities are the same. Differences in other risk markers could explain these differences. Edinburgh men more often had raised serum triglycerides, more often smoked, and had higher systolic blood pressures. Compared with the Scots, more than twice as many of the Stockholm men had no high value risk markers.

The emphasis of the report's recommendations is on dietary modification of plasma lipid levels and not on drug treatment. The Coronary Drug Project carried out in the United States clearly showed the small importance of drug treatment both in the reduction of raised blood fats and in lessening of coronary heart disease mortality. ${ }^{4}$ Clofibrate is widely prescribed, but is relatively ineffective in the reduction of hypercholesterolaemia. Raised triglycerides often reflect an excess of carbohydrate calories in the diet (including alcohol) and are more effectively lowered by calorie reduction than by prescription of expensive drugs.

Many of our national foods are "super saturated" right from roast beef and Yorkshire pudding down to bangers and mash, and any serious effort at health education must take account of this. Fish and chips should be all right (they may disappear with the cod war and potato shortage), but they are innocuous only when frying is carried out with a polyunsaturated oil which is not frequently reused. School meals need radical change, for likes and dislikes are largely determined during childhood. Ice-cream made with mutton fat should be replaced with a version made with polyunsaturated fat. No longer should we be urged from the hoardings to "go to work on an egg" or to "drink another pinta." If they are to be chosen by the housewife, polyunsaturated margarines would have to be marketed at prices lower than the price of butter. In Australia there is already trouble with the dairy farmers over eggs and in Britain and the EEC margarine and butter interests have collided over advertising regulations. A change in the national diet unfortunately requires rather more than suggestions from a royal college.

The case against high blood pressure is equally unassailable, but statistics show that treated hypertensives still die from coronary heart disease. ${ }^{5}$ The formation and rupture of Charcot-Bouchard aneurysms on the cerebral arteries is determined by the blood pressure of the moment, so that the incidence of stroke drops once control of blood pressure has been attained; but it seems that coronary artery disease persists as a 
legacy of past years of hypertension. We now know that high blood pressure often starts in childhood, though it may come to light only after several decades of unimpeded onslaught on the arteries. The report urges that the opportunity should be taken to record the blood pressure in every patient coming to the consulting rooms.

The smoking story is intriguing, because despite the major association between smoking and coronary heart disease it is probably not an atherogenic agent: thrombosis may follow nicotine-induced vascular spasm, fatty acid release, and platelet aggregation, particularly in atheromatous arteries. ${ }^{6}$ The report suggests that a reduction in cigarette smoking will require fiscal measures. Despite the controversy over infringement of personal liberties the curtailment of smoking in hospital except in certain segregated areas should surely be enforced.

Ideally general practice should provide the setting for identifying those at high risk from coronary heart disease, and the report does not recommend mass screening for coronary heart disease or for its associated risk factors. The efforts of general practitioners should also be directed towards public health education about diet and the detection of high blood pressure in the young. Though the report suggests that children may share all of the major risk factors found in adult life, this is not often so: raised triglycerides are rarely found until the third decade or later and familial hypercholesterolaemia, affecting only $3 \%$ of the population, is the only cause of high blood fats which is at all common in childhood. Obesity is a risk factor when it leads to hyperlipidaemia, and both regular physical exercise and the lean physique should be sought from childhood on. ${ }^{7}$

Finally, it is reassuring to read that the report does not recommend the discouragement of those qualities of initiative, diligence, leadership, and hard work which on grey days seem to be vanishing attributes.

1 Fournal of the Royal College of Physicians, 1975, 10, 5

2 Keys, A, Fournal of Chronic Diseases, 1966, 19, 245.

3 Oliver, M F, et al, European fournal of Clinical Investigation, 1975, 5, 507.

4 Coronary Drug Project Research Group, fournal of the American Medical Association, 1972, 220, 996.

5 Wilhelmsen, L, Wedel, H, and Tibblin, G, Circulation, 1973, 48, 950.

${ }^{6}$ Rose, G, American Heart fournal, 1973, 85, 838.

7 Morris, J N, et al, Lancet, 1973, 1, 333.

\section{British births 1970}

Few aspects of the nation's health have been examined with such care and in such detail as childbirth. Reports on confidential inquiries into maternal deaths ${ }^{1}$ have helped to make maternity safer for mothers, while the national maternity surveys $^{2-5}$ in 1946,1958 , and 1970 similarly helped to reduce the risks for babies. The recently published report of the 1970 British Births Survey ${ }^{5}$ covered all births in England, Wales, Scotland, and Northern Ireland during the week beginning 5 April 1970.

Many interesting comparisons are made with the study carried out 12 years previously. The perinatal mortality rate fell by about one-third from 33 to 23 per 1000 deliveries, and the reduction was greater among stillbirths $(39 \%)$ than among first week deaths $(14 \%)$. This welcome improvement came largely from a fall in the number of deaths due to pneumonia, birth trauma, pulmonary haemorrhage, and haemolytic disease. But low birthweight remains an obstinate and impor- tant underlying cause of perinatal mortality $-68 \%$ of the babies who died weighed less than $2500 \mathrm{~g}$ at birth.

A complex interplay of medical and social factors underlies these changes in perinatal death rates. More babies were born under specialist supervision in hospitals in 1970, and this included good (though improvable) standards of paediatric care during the first, critical week of life. Some babies were still being sent home from hospital only to be readmitted with clinically substantial problems shortly afterwards. A more comprehensive system of examination of the neonate might have identified babies likely to develop hyperbilirubinaemia or some forms of cerebral irritation. Relatively few babies in 1970 had serum bilirubin, blood sugar, or blood calcium estimations done, and there appeared to be a lack of awareness of the danger of hypothermia. There have doubtless been improvements over the past few years.

During the interval between the 1958 and 1970 surveys there was a drop in the average age of the mothers, with the proportion over 35 years reduced by a third. In some measure the advantage of fewer elderly mothers was offset by the rise in the number of mothers under the age of 20 -there were half as many again as in 1958, and the perinatal death rate in babies born to these young mothers is significantly higher than among women in the age group 20-30 years. It is always difficult in large, national studies to obtain accurate answers about marital status, but there is no doubt that between 1958 and 1970 illegitimacy became less of a social stigma and the rise from 2.9 to $5.5 \%$ (probably an underestimate) indicates another area of maternity where mothers do less well and tend to hold back improvement in the national perinatal mortality rate. In the 1970 study the perinatal mortality rates for married and unmarried mothers were 20.2 and $36.4 \%$ respectively.

A major change in recent years has been the admission of large numbers of young immigrants from countries with different cultural patterns from our own. In 1970 about $11 \%$ of mothers were born outside the United Kingdom and they were more likely to lose their babies. But their relatively high perinatal mortality rate was swamped by the much larger group of mothers born in the United Kingdom. The maternal perinatal mortality rate was raised by only 0.1 per 1000 births by this immigrant group. Depending upon their country of origin some immigrants did worse than others, and this situation is well worth close and continuing study.

The steep social class gradient in perinatal mortality rates persisted in 1970 , ranging from 7.5 in the upper to 27.6 in the lowest socioeconomic group. It is disturbing to find that social class differences in reproductive efficiency are widening rather than diminishing. This particular problem was examined carefully in Aberdeen by Illsley, ${ }^{6}$ who showed that selective interchange between social classes and marriage tends to accentuate class differences. Women who rise in social status at marriage tend to be of superior intelligence, education, and occupational skill and are likely to be taller and in good health; these women are less likely to lose their babies at birth. On the other hand, women who move to a lower socioeconomic status at marriage tend to have the opposite characteristics. This process of selective movement up and down the socioeconomic scale is at least partly responsible for the continuing, even increasing, disparity in the reproductive performances of mothers in the upper and lower social groups.

An important feature of the 1958 survey was the identification of the added risk to babies whose mothers smoked during pregnancy. In spite of all the publicity and propaganda that followed this finding there is little evidence that the smoking habits of pregnant women have changed over the intervening 